\title{
Probability densities of homogeneous functions: explicit approximation and applications to percolating networks
}

\author{
Raphael Blumenfeld \\ School of Physics and Astronomy, Raymond and Beverly Sackler Faculty of Exact Sciences, \\ Tel Aviv University, Tel Aviv 69978, Israel
}

Received 6 July 1987, in final form 30 September 1987

\begin{abstract}
We treat the probability density of homogeneous functions of independent variables. Assuming these variables are narrowly distributed, we find an explicit approxi. mate form for the Laplace transform of the function's density. This expression is used to show that, if the variables are all Gaussian, then so is the global function. The latter result is also claimed to hold for the total resistance of any random resistor network between two arbitrary terminals. We further utilise our result to explicitly find the Laplace transform of the conductance density of (i) a hierarchical structure and (ii) a random network in $1+\varepsilon$ dimensions using the Migdal-Kadanoff procedure. In the last case we also find the scaling of all the cumulants and find that they exhibit a constant gap.
\end{abstract}

\section{Introduction}

Random systems are ones with an inhomogeneous structure. They have been the subject of increasing interest in recent years. Such systems are, for example, diluted resistor networks, porous media and fractured rocks. On top of this geometrical randomness there can be another one. The elementary building blocks of the system may (and usually do) have properties that are distributed with a probability density function (PDF). Therefore even systems that have an identical microstructure reflect this elementary randomness by exhibiting a global PDF of macroscopic properties. One of the fruitful models to treat geometrical randomness is the percolation model $[1,2]$. The random system is idealised to be constructed of two components with concentrations $p$ and $1-p$, respectively. Near the critical concentration $p_{\mathrm{c}}$ (when one of the components forms a continuous path from one boundary to its opposite) many quantities diverge with power laws like $\left|p-p_{c}\right|^{-x}$, where $x$ is called a critical exponent. Many techniques were employed to probe these exponents. Two such techniques relevant to our discussion are the renormalisation group $[3,4]$ and expansion in low dimensions [5].

The first part of this paper treats random systems whose elements are narrowly distributed around some mean value. Assuming the quantity to be renormalised (e.g. the coupling constant between spins, the elastic constant, the dielectric constant or the electrical conductance between two terminals) is an sth-order homogeneous function of the microscopic variables, we find a simple approximate expression for the Laplace transform (LT) of the global PDF. Having an explicit form for the density function enables one to probe details that are finer than just the behaviour of the mean or the second moment. One can, for example, study its tails in order to gain insight on 
processes that are dominated by these tails. Moreover, it may give estimates to the amplitudes of various cumulants and not only for their scaling with the length or the concentration. Using the explicit expression that we find, it is shown that if the microscopic variables are real and Gaussian then, to this approximation, so is the global function's density. This last result is claimed to hold for random resistor networks (RRN) as well, where the variables (resistances) may assume only positive real values. Thus if all the resistors in the network have a narrow Gaussian PDF then the total resistance is also Gaussian. We further treat a specific hierarchical structure [6-10] and find the explicit form for the LT of its total conductance PDF at each iteration. In the second part of the paper we apply our result to find the global PDF of the renormalised conductance in $1+\varepsilon$ dimensions, using the Migdal-Kadanoff [11] procedure. This is the first time that the distribution of such homogeneous functions is discussed in such detail. The technique itself is applicable to general random functions and not only to RRN near the percolation threshold. We also obtain the scaling of the cumulants of the non-linear conductance $[12,13]$,

$$
I=(g V)^{1 / \alpha} \text {. }
$$

For $\alpha=1$ it has been shown [14-16] that the $m$ th cumulant of the resistance diverges with the length $L$ as $L^{x_{m}}$ where $x_{m}$ is non-linear in $m$. These results were extended to include non-linear networks obeying (1) [17] and $x_{m}$ was shown to be a convex function of $m$ for all $\alpha$. Wo show that the critical exponent of the $m$ th cumulant, using the Migdal-Kadanoff procedure in $1+\varepsilon$ dimensions, depends linearly on $m$ for all $\alpha$, in sharp contrast to the above-mentioned results. The latter technique was very recently used to determine the scaling of the cumulants of non-linear resistance for dimensions not below two [18]. Here we study the conductance cumulants, which amounts to discussing the positive moments and dimensions very near one. The results are similar where both approximations overlap.

\section{The global LT}

Assume a function $\Phi$ of $N$ independent and random positive variables, $\sigma_{j}$, i.e. a homogeneous function of sth order in all of them. Each variable has a narrow PDF $f_{j}\left(\sigma_{j}\right)$. Being of $s$ th order, $\Phi$ obeys

$$
\Phi\left(\lambda \sigma_{1}, \lambda \sigma_{2}, \ldots, \lambda \sigma_{N}\right)=\lambda^{s} \Phi\left(\sigma_{1}, \sigma_{2}, \ldots, \sigma_{N}\right)
$$

with $\lambda$ an arbitrary constant. Therefore

$$
s \Phi\left(\sigma_{1}, \sigma_{2}, \ldots, \sigma_{N}\right)=\sum_{j=1}^{N} \sigma_{j}\left(\frac{\partial \Phi}{\partial \sigma_{j}}\right)
$$

which is readily obtained by differentiating both sides of (2) with respect to $\lambda$ at $\lambda=1$. Assuming $f_{J}\left(\sigma_{j}\right)$ is narrow for all $j$ with $\left(\left\langle\delta \sigma_{j}^{2}\right\rangle\right)^{1 / 2} \ll \sigma_{0}=\left\langle\sigma_{j}\right\rangle$, let us expand $\Phi$ around the point $\sigma_{1}=\sigma_{2}=\cdots=\sigma_{N}=\sigma_{0}$,

$$
\Phi\left(\sigma_{1}, \ldots, \sigma_{N}\right) \simeq \Phi_{0}+\sum_{j=1}^{N} g_{j} \delta \sigma_{j}
$$

where $\Phi_{0}=\Phi\left(\sigma_{0}, \ldots, \sigma_{0}\right)$ and $g_{\jmath}=\left[\partial \Phi / \partial \sigma_{j}\right]_{\sigma_{0}}$. Applying (3) yields

$$
\Phi\left(\sigma_{1}, \ldots, \sigma_{N}\right) \simeq(1-s) \Phi_{0}+\sum_{j=1}^{N} g_{j} \sigma_{j} .
$$


The PDF of $G=\Phi\left(\sigma_{1}, \ldots\right)$ is

$$
F(G)=\int_{0}^{x} \delta\left\{G-\Phi\left(\sigma_{1}, \ldots\right)\right\} \prod_{j=1}^{N}\left\{f_{j}\left(\sigma_{j}\right) \mathrm{d} \sigma_{j}\right\} .
$$

Defining $L_{j}\left(k_{j}\right)$ as the LT of $f_{j}\left(\sigma_{j}\right)$, substituting the inverse transform for $f_{j}\left(\sigma_{j}\right)$ and writing the $\delta$ function in an integral form over a variable $K$ yields

$$
\begin{aligned}
F(G)=\left(\frac{1}{2 \pi \mathrm{i}}\right)^{N+1} \int_{-\mathrm{i} x}^{\mathrm{ix}} \mathrm{d} K \exp [K(G-\Phi)] \\
\times \prod_{j=1}^{N}\left(\int_{-\mathrm{i} x}^{\mathrm{ix}} \mathrm{d} k_{j} L_{j}\left(k_{j}\right) \int_{0}^{x} \exp \left(k_{j} \sigma_{j}\right) \mathrm{d} \sigma_{j}\right) .
\end{aligned}
$$

Substituting (5) for $\Phi$ and integrating over the $k$, and the $\sigma_{j}$ yields

$$
F(G) \simeq \frac{1}{2 \pi \mathrm{i}} \int_{-\mathrm{i} x}^{\mathrm{i} \infty} \exp (K \tilde{G})\left(\prod_{j=1}^{N} L_{i}\left(g_{j} K\right)\right) \mathrm{d} K
$$

where $\tilde{G}=G-(1-s) \Phi_{0}$. Now defining

$$
L(K) \equiv \prod_{j=1}^{N} L_{l}\left(g_{j} K\right)
$$

we notice that $L(K)$ is exactly the $\mathrm{LT}$ of $\tilde{F}(\tilde{G})=F(G)$. Following a similar procedure with Fourier transforms, $\gamma_{j}\left(k_{j}\right)$, renders an analogous result, i.e. defining

$$
\Gamma(K) \equiv \prod_{j=1}^{N} \gamma_{j}\left(g_{j} K\right)
$$

then $\Gamma(K)$ is the Fourier transform of $\tilde{F}(\tilde{G})=F(G)$.

We note that results $(9 a)$ and $(9 b)$ also hold for the less constrained variables $-\infty<\sigma_{j}<\infty$. These closed form expressions for the LT and the Fourier transform of the total density function are much easier to treat than (6). Therefore they may render a solution for the global PDF much more readily.

In order to apply (9) to RRN we first note that the conductance (resistance) of the whole network is a first-order homogeneous function of all the individual conductances (resistances), i.e. $s=1$. Thus the global LT is

$$
L(K)=\prod_{j=1}^{N} L_{j}\left(g_{j} K\right)=\int_{0}^{\infty} F(G) \exp (-K G) \mathrm{d} G .
$$

The prefactors $g_{j}$ are $\left(i_{j} \Phi_{0} / \sigma_{0}\right)^{2}$ (or $\left.i_{j}^{2}\right)[16,19]$ for the global PDF of the conductance (resistance) where $i_{j}$ is the fraction of the total current, between the terminals, that is carried by the $j$ th bond in the unperturbed state (i.e. $\sigma_{j}=\sigma_{0}$ for all $j$ ).

The explicit form of the LT enables one to calculate the moments and the cumulants of the global PDF. We recall that the $m$ th cumulant is the prefactor of $(\mathrm{i} k)^{m} / m$ ! in the expansion of the logarithm of the Fourier transform of $F(G), \Gamma(K)$. Employing (9b) we obtain

$$
\ln \Gamma(K)=\sum_{j=1}^{N} \ln \gamma_{j}\left(g_{j} K\right)
$$

Hence the renormalised prefactor is

$$
\left\langle G^{m}\right\rangle_{c}=\sum_{j=1}^{N} g_{j}^{m}\left\langle\sigma_{j}^{m}\right\rangle_{c}
$$


In RRN with identically distributed conductances, (11) simplifies via using Cohn's theorem [19],

$$
\frac{\left\langle G^{m}\right\rangle_{\mathrm{c}}}{\left\langle\sigma^{m}\right\rangle_{\mathrm{c}}}=\sum_{j=1}^{N}\left(\frac{\Phi_{0}}{\sigma_{0}} i_{j}\right)^{2 m}
$$

For non-linear networks obeying (1) $[12,13]$, equation $(12 a)$ generalises to [17]

$$
\frac{\left\langle G^{m}\right\rangle_{\mathrm{c}}}{\left\langle\sigma^{m}\right\rangle_{\mathrm{c}}}=\sum_{j=1}^{N}\left(\frac{\Phi_{0}}{\sigma_{0}}\right)^{2 m}\left(i_{j}\right)^{m(\alpha+1)}
$$

\section{Applications}

\subsection{Narrow Gaussian variables and RRN}

First let us consider a function whose variables have a narrow Gaussian distribution,

$$
f_{j}\left(\sigma_{j}\right)=\frac{1}{(2 \pi)^{1 / 2} \delta_{j}} \exp \left[-\left(\sigma_{j}-\sigma_{0 j}\right)^{2} / 2 \delta_{j}^{2}\right] \quad-\infty<\sigma_{j}<\infty
$$

where $\delta_{j}=\left(\left\langle\sigma_{j}^{2}\right\rangle_{\mathrm{c}}\right)^{1 / 2}$ and $\sigma_{0 j}$ are the mean and the width of the $j$ th variable, respectively. Being Gaussian, $f_{j}\left(\sigma_{j}\right)$ has only two non-zero cumulants-the first and the second. Turning to the global PDF we find from (11) that it also has only these two cumulants as the only non-vanishing ones. Since knowing all the cumulants of such distributions defines them uniquely [20], then to this approximation $F(G)$ is Gaussian too, i.e.

$$
F(G) \simeq \frac{1}{(2 \pi)^{1 / 2} \Delta} \exp \left[-\left(G-s \Phi_{0}\right)^{2} / 2 \Delta^{2}\right]
$$

where $\Delta^{2}=\Sigma_{j=1}^{N} g_{j}^{2} \delta_{j}^{2}$ and $s \Phi_{0}=\Sigma_{j=1}^{N} g_{j} \sigma_{0 j}$ are the width and the mean of the global $\mathrm{PDF}$, respectively. We proceed to show that, to the above-mentioned approximation, the PDF of the conductance of a RRN is Gaussian if all its elements are Gaussian. Let us write the Gaussian PDF for positive variables

where

$$
f\left(\sigma_{j}\right)=\frac{\sqrt{2} \exp \left[-\left(\sigma_{j}-\sigma_{0}\right)^{2} / 2 \delta^{2}\right]}{\delta \sqrt{\pi}[1+\phi(z)]} \quad 0<\sigma_{j}<\infty
$$

$$
\phi(z) \equiv \frac{2}{\sqrt{\pi}} \int_{0}^{z} \exp \left(-x^{2}\right) \mathrm{d} x \quad z=\frac{\sigma_{0}}{\delta \sqrt{2}} \gg 1 .
$$

The cumulants of each individual conductance are exponentially close to those of (13). For example, the first and second cumulants of (15) are $\left\langle\sigma_{j}\right\rangle=\sigma_{0}(1+\lambda)$ and $\left\langle\sigma_{j}^{2}\right\rangle_{\mathrm{c}}=$ $\delta^{2}-\sigma_{0} \lambda(1+\lambda)$ where $\lambda=\lambda(z)=\exp \left\{-z^{2} /[2 \sqrt{\pi}(1+\phi(z))]\right\}$. Similarly, higher cumulants are exponentially small. Using (11) for the cumulants of $F(G)$ now yields the result that they are exponentially close to those of (14), where $z$ is replaced by $Z=s \Phi_{0} /(\Delta \sqrt{2})$. For large networks we expect $Z$ to be very large so that $F(G)$ approaches the Gaussian form very fast as the network approaches the thermodynamic limit.

We note that the global Gaussian behaviour only reflects the original local Gaussian distribution. The essential behaviour, however, need not necessarily be such. For example, assume that the distribution of the local variables is constant between $1+\Delta$ and $1-\Delta$. The LT in this case is, in our approximation,

$$
L(T)=\exp \left\{-R_{0} T+N\left[\ln \left(\frac{\sinh (g \Delta T)}{g \Delta T}\right)\right]_{\mathrm{av}}\right\}
$$


where the averaging in the exponent is over the distribution of the variables $g$. This $\mathrm{LT}$ is not Gaussian in general. The last example demonstrates that the approximation may well go beyond the Gaussian behaviour.

\subsection{Hierarchical structure}

Let us consider a network of resistors that is constructed by $n$ iterations of a hierarchical structure [10] as shown in figure 1 . Such structures are used to model randomness of geometry $[4,6-9,13]$ because many problems have exact solutions on them. Assume each bond is a resistor of mean resistance $r_{0}$ and cumulants $\left\langle r^{m}\right\rangle_{c}$. After $n$ iterations the structure consists of $C_{j}^{n}$ bonds that support a fractional current of $i_{j}=2^{-j}$ of the total current between the terminals $(j=0,1, \ldots, n)$, where $C_{j}^{n}=n ! /[j !(n-j) !]$. The total number of bonds sums up to $N=4^{n}$. Hence the LT of the global PDF of the non-linear resistance is

$$
L(K)=\prod_{j=1}^{N(n)} L\left(g_{j} K\right)=\left(\prod_{j=1}^{n}\left[L\left(K a^{j}\right)\right]^{C_{j}}\right)^{2^{n}}
$$

where $a=2^{-(\alpha+1)}$ and $\alpha$ is the non-linearity parameter in (1). The width of the total PDF is

$$
\Delta=\delta\left(\sum_{j=1}^{N} g_{j}^{2}\right)^{1 / 2}=\delta\left(\sum_{j=1}^{N} i_{j}^{2(\alpha+1)}\right)^{1 / 2}
$$

or

$$
\Delta=\delta\left[2+2^{-(2 \alpha+1)}\right]^{n / 2}
$$

in accordance with previous results $[10,13]$. The total mean resistance is

$$
R_{0}=r_{0} \sum_{j=1}^{N} g_{j}=r_{0}\left(2+2^{-\alpha}\right)^{n}
$$

In general, the $m$ th cumulant is

$$
\left\langle R^{m}\right\rangle_{\mathrm{c}}=\left\langle r^{m}\right\rangle_{\mathrm{c}} \sum_{j=0}^{n} C_{j}^{n} 2^{n-j(\alpha+1) m}=\left\langle r^{m}\right\rangle_{\mathrm{c}}\left(2+2^{1-m(\alpha+1)}\right)^{n} .
$$

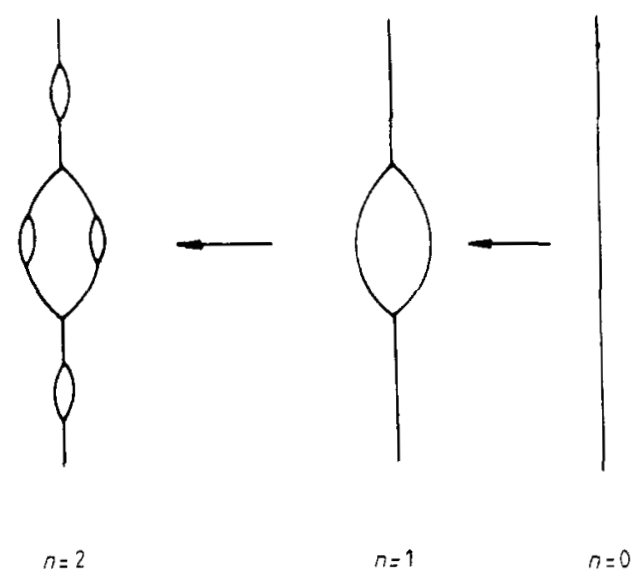

Figure 1. Two iterations of the hierarchical structure. 
The noise in the system is defined by

$$
S_{R}=\frac{\left\langle R^{2}\right\rangle_{\mathrm{c}}}{\langle R\rangle^{2}}=\frac{\delta^{2}}{r_{0}^{2}}\left(\frac{2+2^{-(2 \alpha+1)}}{\left(2+2^{-\alpha}\right)^{2}}\right)^{n}
$$

From (19) we can solve for the total PDF,

$$
\ln (\Gamma(K))=\sum_{j=1}^{\infty} \frac{(\mathrm{i} K)^{m}}{m !}\left(2+2^{1-m(\alpha+1)}\right)^{n}\left\langle r^{m}\right\rangle_{c} .
$$

Hence

$$
F(G)=\frac{1}{2 \pi} \int_{0}^{\infty} \exp \left(\sum_{m=1}^{\infty} \frac{(\mathrm{i} K)^{m}}{m !}\left(2+2^{1-m(\alpha+1)}\right)^{n}\left\langle r^{m}\right\rangle_{c}-\mathrm{i} K G\right) \mathrm{d} K
$$

The application of the treatment to more complicated hierarchical structures is straightforward. Expression (21) is in some sense complementary to the distribution of voltages (or currents) studied in previous works $[10,15,17]$. In [10] it was shown that, for the above-mentioned hierarchical structure, the density of the voltage drop on each individual resistor (in the ohmic case) takes the form of a log-binomial distribution. This log-binomial distribution is (up to a normalising constant) the probability density of the variable $g_{j}$. Thus (8) and (9) establish a correspondence between the density of the currents and that of the global resistance.

\section{The global PDF of conductors in $1+\varepsilon$ dimensions}

Next we apply (9) to find the global PDF of a network in $1+\varepsilon$ dimensions using a Migdal-Kadanoff renormalisation procedure [11] (figure 2). We further generalise this treatment to non-linear conductors obeying (1). The global $V-I$ characteristic plot of such a network also obeys (1) $[12,13]$. Therefore the total conductance of the
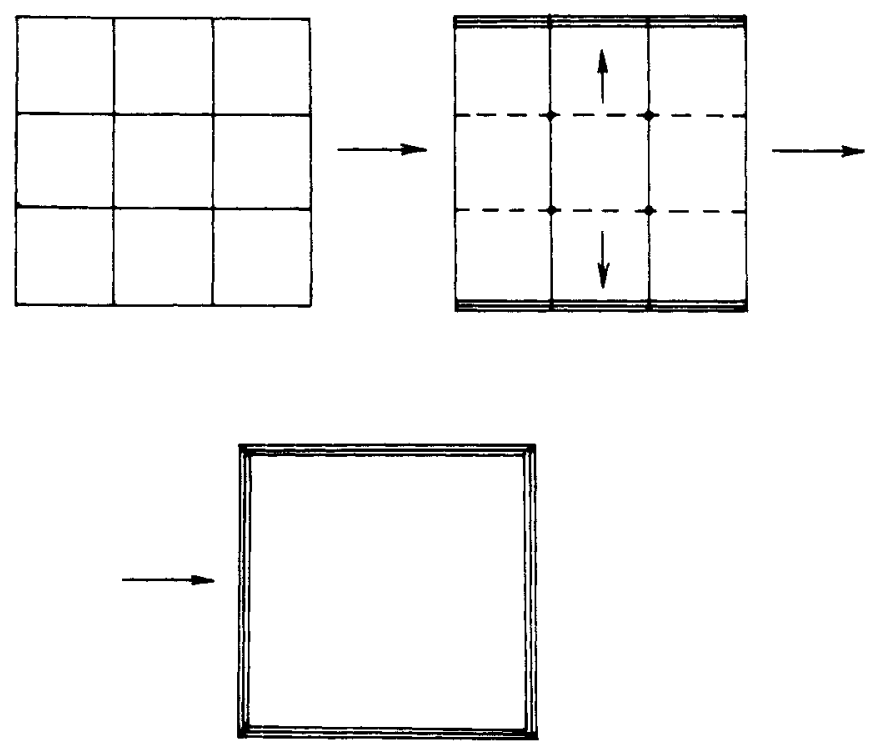

Figure 2. Migdal-Kadanoff renormalisation procedure in two dimensions. 
network is a first-order homogeneous function of the individual conductances. Consequently, it obeys (2) and (3) with $s=1$, thus lending itself to our treatment. Kirkpatrick [5] used this procedure to study the first moment of the total linear conductance. We extend this model for the global PDF and for all the cumulants of linear and non-linear such networks.

The procedure consists of finding the effective non-linear conductance of $u=b^{d-1}$ bonds in parallel $\left[R_{\mathrm{p}}(b)\right]^{u}$ and then finding the conductance of $b$ such constructions in series, $R_{\mathrm{s}}(b)$. The total conductance is

$$
G_{\mathrm{T}}=\left\{R_{\mathrm{s}}(b)\left[R_{\mathrm{p}}(b)\right]^{u}\right\} g
$$

where $g$ is the conductance of an individual bond, and $R_{\mathrm{s}}$ and $R_{\mathrm{p}}$ should be understood as operators. The quantities $b$ and $d$ are treated as continuous parameters. For $d=1+\varepsilon$ and $b=1+\eta$ ( $\varepsilon$ and $\eta$ being much smaller than one) equation (22) can be linearised to yield

$$
\left\langle G_{\mathrm{T}}\right\rangle /\langle g\rangle=1+\eta\left(\Lambda_{\mathrm{s}}+\varepsilon \Lambda_{\mathrm{p}}\right)
$$

where $\Lambda_{\mathrm{s}}$ and $\Lambda_{\mathrm{p}}$ are the series and parallel infinitesimal transformations. A similar procedure for the $m$ th cumulant $\psi(m, \alpha)$ yields

$$
\left\langle G_{\mathrm{r}}^{m}\right\rangle_{\mathrm{c}} /\left\langle g^{m}\right\rangle_{\mathrm{c}}=1+\eta\left(\Lambda_{\mathrm{s}}^{(m)}+\varepsilon \Lambda_{\mathrm{p}}^{(m)}\right) .
$$

The critical exponent of the $m$ th cumulant $\psi(m, \alpha)$ is defined through

$$
\left\langle G_{\mathrm{T}}^{m}\right\rangle_{\mathrm{d}} /\left(g^{m}\right\rangle_{\mathrm{c}}=b^{\psi(m, a)-2 m \psi(1, \alpha)}=1+\eta[\psi(m, \alpha)-2 m \psi(1, \alpha)] .
$$

Comparing (25) to $(24)$ yields

$$
\psi(m, \alpha)=\Lambda_{\mathrm{s}}^{(m)}+\varepsilon \Lambda_{\mathrm{p}}^{(m)}+2 m \psi(1, \alpha) .
$$

The $m$ th cumulant of the conductance of a parallel blob constructed of $u=b^{d-1}$ bonds is

$$
\left\langle g_{\mathrm{p}}^{m}\right\rangle_{\mathrm{c}}=\frac{\sum_{n=1}^{u} C_{j}^{n} p_{\mathrm{c}}^{n} q_{\mathrm{c}}^{u-n}\left\langle g_{n}^{m}\right\rangle_{\mathrm{c}}}{1-q_{\mathrm{c}}^{u}}
$$

where $\left\langle g_{n}^{m}\right\rangle_{c}$ is the $m$ th cumulant of the conductance of the $n$-parallel blob and $q_{\mathrm{c}}=1-p_{\mathrm{c}}$. Using (11) we derive

$$
\left\langle g_{n}^{m}\right\rangle_{\mathrm{c}}=\left\langle g^{m}\right\rangle_{\mathrm{c}} n^{1+m(\alpha-1)} .
$$

Inserting (28) into (27) we note that the sum can be readily evaluated. This exercise is performed in appendix 1 to yield

$$
\left\langle g_{\mathrm{p}}^{m}\right\rangle_{\mathrm{c}} /\left\langle\mathrm{g}^{m}\right\rangle_{\mathrm{c}} \approx 1+\eta \varepsilon[1+m(\alpha-1)]
$$

Thus we identify

$$
\Lambda_{\mathrm{p}}^{(m)}=1+m(\alpha-1) .
$$

In order to find $\Lambda_{\mathrm{s}}^{(m)}$ we use (11) and the fact that non-linear conductances defined by (1) combine in series just as the linear ones. Hence

$$
\left\langle g_{\mathrm{s}}^{m}\right\rangle_{\mathrm{c}} /\left\langle g^{m}\right\rangle_{\mathrm{c}}=b^{1-2 m} \simeq 1+\eta(1-2 m)
$$

or

$$
\Lambda_{\mathrm{s}}^{(m)}=1-2 m .
$$


Combining (26), (30) and (31) yields

$$
\psi(m, \alpha)=1+\varepsilon[1-m(\alpha+1)] .
$$

We note that $\psi(1, \alpha)$ which is the critical exponent of the non-linear conductance is a monotonically decreasing function of $\alpha$ as expected [13]. For $\alpha \rightarrow \infty$ we see that the total conductance vanishes as expected for a structure containing no singly connected bonds $(\varepsilon \rightarrow 0)$, again in agreement with the results in [13]. However, in order that the above treatment remain valid, the quantity $k=1+m(\alpha-1)$ should be positive. Another interesting feature of equation (32) is that it displays a constant gap of $\varepsilon(\alpha+1)$ between any two successive exponents. This property stems from the linearisation procedure itself that is performed in appendix 1 and will be commented upon in the concluding section. After completing this section we learnt that results for non-linear networks using this technique were recently obtained for the resistance cumulants [18]. However, there the negative moments of the sum (27) were treated. The solution technique depends strongly on the regime of moments probed and influences the results. Nevertheless, in the region where the two approximations overlap the results are similar apart from the fact that we ignored terms of order $\exp (-1 / \varepsilon)$ relative to $\varepsilon$.

In order to find the global PDF, $F\left(G_{\mathrm{T}}\right)$, we have to obtain the global LT, $L_{\mathrm{T}}(K)$. We do this in appendix 2 and find

$F\left(G_{\mathrm{T}}\right) \simeq(1-\alpha \eta) f\left(G_{\mathrm{T}}\right)+\frac{\eta}{2 \pi \mathrm{i}} \int_{-i \infty}^{\mathrm{i} \infty} \exp \left(K G_{\mathrm{T}}\right) L(K)\left\{\alpha K G_{\mathrm{T}}+(1+\varepsilon) \ln [L(K)]\right\} \mathrm{d} K$

where $f(g)$ and $L(K)$ are the PDF of a single bond's conductance and its LT, respectively. In the limit $\eta \rightarrow 0,\left(G_{\Upsilon}\right)$ degenerates into $f(g)$ as it should. To the best of our knowledge this is the first time a simple expression for the density function of the conductance has been presented.

One can now analyse it, e.g. obtain its tail behaviour. We will treat this problem elsewhere.

\section{Conclusions}

To conclude we have derived the PDF of an sth-order homogeneous function of random variables, to the lowest order in the relative widths of the individual PDF. Since many properties of systems in nature can be described by such functions we believe that (9) may have many applications. We used (9) to show that a function of narrow Gaussian independent random variables remains Gaussian with rescaled parameters $\Delta$ and $G_{0}$. We have claimed and demonstrated that this result also holds for RRN up to exponentially small corrections. We considered a specific hierarchical structure that models RRN at the percolation threshold and found the explicit form of the Laplace transform of its conductance PDF. Next we have found the PDF of the RRN in $1+\varepsilon$ dimensions by the Migdal-Kadanoff renormalisation group approach. In this model we considered non-linear conductors and calculated the critical exponent, $\psi(m, \alpha)$ of the $m$ th cumulant of the total non-linear conductance. This exponent was found to depend linearly on $m$ and on $\alpha$ which implies a constant gap between successive exponents. A parallel result was obtained recently for the resistance cumulants [18]. Our result differs from the latter because in our case terms of order $\exp (-1 / \varepsilon)$ are negligible in relation to terms of order $\varepsilon$. The constant gap, however, stems from the linearisation 
procedure that is inherent to the expansion in $\varepsilon$. Therefore we conclude that this approximation is correct for small values of $\varepsilon$ but cannot be extrapolated successfully for dimensions above two. In that regime the method used in [18] should be used. For $m=\alpha=1$ our results coincide with previous ones [5].

Finally, we emphasise that the approximation assumes

$$
\left(\left\langle\delta \sigma^{2}\right\rangle\right)^{1 / 2} \ll \sigma_{0}=\langle\sigma\rangle^{2} .
$$

Whenever this condition is not met one has to consider higher and higher terms in equation (4) leading to a totally different form of equations (9).

\section{Acknowledgments}

I am grateful to Professor A Aharony for his critical reading of this work and for helpful suggestions. I am also indebted to Professor A-M S Tremblay for helpful discussions and for clarifying the results in [18]. This work was supported by grants from the US-Binational Science Foundation (BSF) and the Israeli Academy of Science and Humanities.

\section{Appendix 1}

Let us first evaluate the sum in the numerator of equation (27):

$$
I_{k}=\sum_{j=1}^{u} C_{n}^{u} p_{\mathrm{c}}^{n} q_{\mathrm{c}}^{u-n} n^{k}
$$

where $k=1+m(\alpha-1)$. The quantity $n^{k}$ can be written as a sum of factorials [21],

$$
n^{k}=\sum_{j=1}^{k+1}{ }^{j} \sigma_{k}(n)_{j}
$$

where ${ }^{j} \sigma_{k}$ is the Stirling number of the second kind and $(n)_{j}$ is the factorial

$$
(n)_{j}=n(n-1) \ldots(n-j+1) \text {. }
$$

Inserting (A1.3) into (A1.1) and summing over $n$ yields

$$
I_{k}=\sum_{j=1}^{k+1}{ }^{j} \sigma_{k} p_{c}^{j}(u)_{j} .
$$

Remembering that [5]

$$
p_{\mathrm{c}}=1-\theta \quad \theta=\exp (-1 / \varepsilon)
$$

and using (A1.4) one can expand to linear power in $\theta$ to find

$$
\begin{gathered}
H=\left\langle g_{\mathrm{p}}^{m}\right\rangle_{\mathrm{c}} /\left\langle\mathrm{g}^{m}\right\rangle_{\mathrm{c}} \equiv \Lambda_{\mathrm{p}} \simeq\left(1+\theta^{u}\right) \sum_{j=1}^{k+1} \sigma_{k}(u)_{j}(1-j \theta) \\
=\left(1+\theta^{u}\right)\left(u^{k}-\theta \sum_{j=1}^{k+1} j^{j} \sigma_{k}(u)_{j}\right)
\end{gathered}
$$

The next step is to evaluate the sum within the large brackets. Defining

$$
J_{k}(u)=\sum_{j=1}^{k+1} j^{\jmath} \sigma_{k}(u)_{j}
$$


utilising the definition of the difference of this sum [21],

$$
\Delta\left(y^{k}\right)=\sum_{j=1}^{k+1} j^{\jmath} \sigma_{k}(y)_{j-1}
$$

replacing $y$ by $x-1$ and using the identity

$$
(x-1)_{n-1}=(x)_{n} / x
$$

yields in (A1.7)

$$
\Delta\left((x-1)^{k}\right)=\frac{1}{x} \sum_{j=1}^{k+1} j^{j} \sigma_{k}(x)=J_{k}(x) / x
$$

Employing $\Delta\left((x-1)^{k}\right)=x^{k}-(x-1)^{k}$, which is the definition of the mathematical difference, we obtain

$$
J_{k}(u)=u^{k+1}\left[1-(1-1 / u)^{k}\right] .
$$

Inserting (A1.10) into (A1.6), expanding $u$ in powers of $\eta$ and maintaining only the linear term yields

$$
H=1+k \eta \varepsilon+\theta \times \begin{cases}-\eta(1+\varepsilon) & k>1 \\ \eta^{k} \varepsilon^{k} & k<1 \\ -\eta & k=1 .\end{cases}
$$

Neglecting now terms of order $\theta$ with respect to $\varepsilon$ we finally obtain equation (29) in the text.

\section{Appendix 2}

First let us find the Laplace transform (LT) of the PDF of a blob of $u=b^{d-1}$ parallel bonds, $L_{\mathrm{p}}(K)$

$$
L_{\mathrm{p}}(K)=\frac{1}{1-q_{\mathrm{c}}^{u}} \sum_{n=1}^{u} C_{n}^{u} p_{\mathrm{c}}^{n} q_{\mathrm{c}}^{u-n} L^{n}(K)=\frac{\left(q_{\mathrm{c}}+p_{\mathrm{c}} L(K)\right)^{u}}{1-q_{\mathrm{c}}^{u}} .
$$

Expanding (A2.1) to first order in $\eta$ and $\varepsilon(\eta=b-1 \ll 1, \varepsilon=d-1 \ll 1)$, recalling that $p_{\mathrm{c}}=1-\exp (-1 / \varepsilon)$ and discarding terms of order $\exp (-1 / \varepsilon)$ with respect to $\varepsilon$, we obtain

$$
L_{\mathrm{p}}(K)=L(K)[1+\eta \varepsilon \ln L(K)] .
$$

Now the global LT of the total PDF is

$$
L_{\mathrm{T}}(K)=\prod_{j=1}^{b} L_{\mathrm{p}}\left(g_{j} K\right)=\prod_{j=1}^{b} L_{\mathrm{p}}\left(K b^{-\alpha}\right) .
$$

Expanding again to first order in $\eta$ and substituting for $L_{\mathrm{p}}(K)$ from (A2.2) yields

$$
L_{\mathrm{T}}(K) \simeq L(K)+\eta\left[(1+\varepsilon) L(K) \ln L(K)-\alpha K L^{\prime}(K)\right]
$$

where $L^{\prime}(K)=[\partial L(x) / \partial x]_{x=K}$. Performing the inverse transform yields for the global PDF

$F(G) \simeq(1-\eta \alpha) f(G)+\frac{\eta}{2 \pi \mathrm{i}} \int_{-i \infty}^{\mathrm{i} x} \exp (K G) L(K)[(1+\varepsilon) \ln L(K)+\alpha K G] \mathrm{d} K$

which is the expression given in the text. 


\section{References}

[1] Stauffer D 1979 Phys. Rep. 541

[2] Essam J W 1980 Rep. Prog. Phys. 43843

[3] Niemeijer T and van Leeuwen J M J 1974 Physica 7117

Burkhardt T W and van Leeuwen J M J (ed) 1982 Real Space Renormalization (Berlin: Springer)

[4] Stinchcombe R B and Watson B P 1976 J. Phys. C: Solid State Phys. 93221

[5] Kirkpatrick S 1977 Phys. Rev. B 151533

[6] Bernasconi J, Schneider W R and Weisman M J 1977 Phys. Rev. B 165250

[7] Berker A N and Ostlund S 1979 J. Phys. C: Solid State Phys. 124961

[8] Kaufman M and Griffiths R B 1981 Phys. Rev. B 24496

[9] Mandelbrot B B and Given J A 1984 Phys. Rev. Lett. 521853

[10] de Arcangelis L, Redner S and Coniglio A 1985 Phys. Rev. B 314725

[11] Migdal A A 1975 Zh. Eksp. Teor. Fiz. 691457 (Sov. Phys.-JETP 42 743) Kadanoff L P 1976 Ann. Phys., NY 100359

[12] Kenkel S W and Straley J P 1982 Phys. Rev. Lett. 49767 Straley J P and Kenkel S W 1984 Phys. Rev. B 296299

[13] Blumenfeld R and Aharony A $1985 \mathrm{~J}$. Phys. A: Math. Gen. 18 L443

[14] Rammal R 1985 J. Physique Lett. 46129

[15] Rammal, R, Tannous C and Tremblay A-M S 1985 Phys. Rev. A 312662

[16] Rammal R, Tannous C, Breton P and Tremblay A-M S 1985 Phys. Rev. Lett. 541718

[17] Blumenfeld R, Meir Y, Aharony A and Harris A B 1987 Phys. Rev. B 353524

[18] Rammal R and Tremblay A-M S 1987 Phys. Rev. Lett. 58415

[19] Cohn R M 1950 Am. Math. Soc.j1 316

[20] Feller W 1951 An Introduction to Probability Theory and its Applications (New York: Wiley)

[21] Jordan K 1950 Calculus of Finite Differences (New York: Chelsea) 